\title{
Review
}

\section{Immunoassay-Based Drug Tests Are Inadequately Sensitive for Medication Compliance Monitoring in Patients Treated for Chronic Pain}

Marion L. Snyder, PhD, Corinne R. Fantz, PhD, and Stacy Melanson, MD, PhD

From: Brigham and Women's Hospital, Boston, MA

Address Correspondence: Stacy Melanson, MD, PhD Brigham and Women's Hospital 75 Francis Street Amory 2

Boston, MA 02115 E-mail:

semelanson@partners.org

Disclaimer: There was no external funding in the preparation of this manuscript.

Conflict of interest: Each

author certifies that he or

she, or a member of his or her immediate family, has no

commercial association (i.e., consultancies, stock ownership, equity interest, patent/licensing arrangements, etc.) that might

pose a conflict of interest in connection with the submitted manuscript.

Manuscript received: o8-01-2015 Revised manuscript received: 12-01-2015

Accepted for publication: 01-12-2016

Free full manuscript: www.painphysicianjournal.com
Background: Enzyme immunoassays (EIA) have notable limitations for monitoring therapeutic compliance in pain management. Chromatography coupled with mass spectrometry provides definitive results and superior sensitivity and specificity over traditional EIA testing.

Objective: To analyze and compare the sensitivity of EIA results together with known prescriptions to liquid chromatography-tandem mass spectrometry (LC-MS/MS) for monitoring drug use (and abuse) in patients treated for chronic pain.

Study Design: A total of 530 urine samples from patients being treated for chronic pain were studied.

Setting: Pain management clinic in the United States.

Methods: The samples were tested for a profile of chronic pain medications and illicit drugs with commercially available EIA kits followed by analysis with Agilent LC-MS/MS system.

Results: The ElAs exhibited poor sensitivity and high rates of false negative results in the pain management setting. For example, $21 \%$ of EIA for opiates show false negative results. Mass spectrometry methods were more sensitive, detected a broader range of drugs and metabolites, and could detect non-prescribed drug use and simulations in compliance.

Limitations: Patients do not always accurately report drug use information, and some drugs do not have EIA methods available for comparative purposes.

Conclusions: Mass spectrometry is a more robust and reliable method for detection of drugs used in the pain management setting. Due to the extent of undisclosed use and abuse of medications and illicit drugs, LC-MS/MS testing is necessary for adequate and accurate drug detection. In addition, LC-MS/MS methods are superior in terms of sensitivity and number of compounds that can be screened, making this a better method for use in pain management.

Key words: Pain management, enzyme immunoassays, mass spectrometry, urine drug testing, prescription status, compliance

Pain Physician Opioid Special Issue 2017; 20:SE1-SE9
D isk stratification for patients prescribed controlled substances is an important part of the management of patients with chronic pain. However, targeted testing based on risk stratification using subjective and objective tools has a number of limitations, including misclassifying patients as compliant or noncompliant with their therapeutic regimen (1-13). Therefore, guidelines from pain management societies state that urine drug testing (UDT) should be used as an additional 
tool to monitor compliance (14). Societal guidelines for UDT recommend both enzyme immunoassay (EIA) and confirmatory/definitive testing by a more sensitive and specific technique such as gas chromatographymass spectrometry (GC-MS) or liquid chromatographytandem mass spectrometry (LC-MS/MS). However, specific testing algorithms and guidelines are not provided; in particular, clinical situations and/or drug classes for which EIA and/or LC-MS/MS should be utilized are not adequately outlined.

EIA screening has known limitations for monitoring compliance in pain management (15-22). The factor that most limits EIA utility in pain management is the lack of EIA sensitivity for detecting specialty or designer drugs, as well as commonly prescribed drugs and illicit drugs at concentrations often present in patients treated for chronic pain. In addition, EIA screening is not available for a number of drugs of interest. Numerous published studies demonstrate high rates of false negatives by EIA in samples originating from patients treated for chronic pain for many important drugs/drug classes, including amphetamines (false negative rates, depending on EIA used and sample population: 28 $60 \%)$, benzodiazepines (18 - 53\%), THC (38\%), cocaine $(42-75 \%)$, methadone $(4-10 \%)$, opiates/oxycodone (8 $-70 \%)$, and propoxyphene (28\%), among others $(15,19,23-25)$.

In an attempt to overcome the limitations of EIA, some private party and government insurances have proposed EIA screening followed by LC-MS/MS if the screen is positive, and also if the screen is unexpectedly negative for a medication that the patient is prescribed (termed 'pertinent negative') (26). There is a lack of clinical data showing the sensitivity of this testing algorithm for monitoring medication compliance in patients treated for chronic pain. Reflexing pertinent negatives to LC-MS/MS should reduce the rate of EIA false negatives (i.e., drugs present at urinary concentrations below the EIA cutoffs and/or are poorly detected by EIA) in patients treated for chronic pain. However, importantly patients screening falsely negative by EIA due to undisclosed medication use would be missed. The goal of this study was to show the sensitivity of this testing algorithm compared to LCMS/MS, the gold standard, and make testing recommendations based on these findings.

\section{Methods}

A total of 530 urine samples from patients being treated for chronic pain were collected. All samples were analyzed upon receipt in the laboratory (LabSource LLC, Greenville, SC). Table 1 outlines the testing performed and corresponding cutoffs utilized. Urine creatinine was measured on all urine samples. Patients' prescribed medications were collected from the test requisition where the provider lists the patients' medications at the time of testing.

\section{Immunoassay}

EIA (Lin-Zhi International, Sunnyvale, CA) was used to test for buprenorphine. Diagnostics Reagents Incorporated (DRI) assays, manufactured by Thermo Scientific (formerly Microgenics, Fremont, CA), were used for all other compounds. Both Lin-Zhi EIA and DRI methods rely on measurement of a change in the enzymatic activity (change in rate of absorbance increase) after combining a sample with an anti-drug antibody and enzyme-drug conjugate and were tested on Olympus AU640 or AU480 analyzers (Beckman Coulter, Brea, CA) following manufacturers' recommendations. The assay cutoff values $(\mathrm{ng} / \mathrm{mL})$ were opiates: 1000, oxycodone: 300, benzodiazepine: 200, amphetamine: 1000, cocaine: 300 , buprenorphine: 10 .

Thermo Scientific DRI Creatinine-Detect specimen validity test was used for urine creatinine measurement (reference range $20-300 \mathrm{mg} / \mathrm{dL}$ ).

\section{LC-MS/MS}

All 530 samples were tested for the following compounds as shown in Table 1: amphetamines, benzodiazepines/metabolite, buprenorphine (Bup), norbuprenorphine (NBup), benzoylecgonine, carisoprodol/ metabolite, fentanyl/metabolite, methadone/metabolite, meperidine/metabolite, opiates, oxycodone/metabolites, propoxyphene, tapentadol, and tramadol/ metabolite by LC-MS/MS at LabSource, LLC, Greenville, SC, using the following summarized protocol: $0.1 \mathrm{~mL}$ aliquots of urine were prepared with $0.05 \mathrm{~mL}$ of multiple deuterated internal standards and $0.75 \mathrm{~mL}$ HPLC grade water. The prepared samples were hydrolyzed with 0.1 $\mathrm{mL}$ of $4.5 \%$ ß-glucuronidase (Patella vulgata) in $\mathrm{pH} 5.0$ acetate buffer for 2 hours at $60 \circ C$, a routine step for enzymatic hydrolysis of glucuronides in urine.

Samples were analyzed via an Agilent 1200 LC series combined with an Agilent $6410 \mathrm{MS} / \mathrm{MS}$ system equipped with an electrospray ionization (ESI) probe in positive ionization mode. Separation was achieved using an Agilent Poroshell 120 EC-C18, $2.1 \times 100 \mathrm{~mm}$, $2.7 \mu \mathrm{m} 600$ bar analytical column (Agilent, Santa Clara, CA). Two ion transitions were monitored for each an- 
Table 1. Immunoassay (EIA) and LC-MS/MS testing performed and corresponding cutoffs.

\begin{tabular}{|l|c|c|l||}
\hline Drug/Drug Class & $\begin{array}{c}\text { Immunoassay Cutoff } \\
(\mathbf{n g} / \mathbf{m L})\end{array}$ & $\begin{array}{c}\text { LC-MS/MS } \\
\text { Cutoff } \mathbf{( n g / m L )}\end{array}$ & \multicolumn{1}{|c||}{$\begin{array}{c}\text { Drugs and Metabolites Detected by } \\
\text { LC-MS/MS }\end{array}$} \\
\hline Amphetamines & 1000 & 50 & $\begin{array}{l}\text { Amphetamine, MDMA } \\
\text { Methamphetamine }\end{array}$ \\
\hline Benzodiazepines & 200 & 50 & $\begin{array}{l}\text { Alpha-hydoxyalprazolam, 7-Amino-clonazepam, } \\
\text { Alpha-hydroxymidazolam } \\
\text { Lorazepam, Nordiazepam, Oxazepam, Temazepam, } \\
\text { Alpha-hydroxytriazolam }\end{array}$ \\
\hline Buprenorphine & 10 & 100 & $\begin{array}{l}\text { Buprenorphine } \\
\text { Norbuprenorphine }\end{array}$ \\
\hline Carisoprodol & N/A & 10 & Carisoprodol, Meprobamate \\
\hline Cocaine & 300 & 50 & Benzoylecgonine \\
Metabolite & N/A & 2.5 & Fentanyl, Norfentanyl \\
\hline Fentanyl & N/A & 10 & $\begin{array}{l}\text { Normeperidine } \\
\text { Meperidine }\end{array}$ \\
\hline Meperidine & N/A & 50 & Methadone, EDDP \\
\hline Methadone & 1000 & 50 & $\begin{array}{l}\text { Codeine, Morphine, Hydrocodone, Hydromorphone, } \\
\text { Norhydrocodone }\end{array}$ \\
\hline Opiates & 300 & 50 & Oxycodone, Oxymorphone, Noroxycodone \\
\hline Oxycodone & N/A & 50 & Propoxyphene \\
\hline Propoxyphene & N/A & 10 & Tapentadol \\
\hline Tapentadol & N/A & 50 & Tramadol, O-desmethyl-tramadol \\
\hline Tramadol & & &
\end{tabular}

$\mathrm{N} / \mathrm{A}=$ not performed

Note: Creatinine was also measured in all urines (reference range $=20-300 \mathrm{mg} / \mathrm{dL}$ ). Buprenorphine was measured by EIA from Lin-Zhi International; all other compounds were measured by EIA from Diagnostics Reagents Incorporated (DRI), Thermo Scientific.

alyte and one transition for each internal standard in dynamic multiple reaction monitoring mode (dMRM). Each analytical run was independently calibrated and relative retention times were used to identify unknown analytes in patient specimens.

A drug-free negative urine control was used to internally prepare controls containing all measured drugs/ metabolites and establish limits of an acceptable range of $\pm 20 \%$. A purchased control containing buprenorphine, amphetamine, methamphetamine, benzoylecgonine, methadone, morphine, oxazepam, oxycodone, and propoxyphene (Stat-Skreen 2X, Biochemical Diagnostics) with an acceptable range of $20 \%$ was also included with each run. A hydrolysis check solution containing $100 \mathrm{ng} / \mathrm{mL}$ of morphine 3-ß-glucuronide was also added to each run to monitor the efficiency of the hydrolysis step with an acceptable free morphine recovery of $70 \mathrm{ng} / \mathrm{mL} \pm 15 \%$. Patient results were not reported if any of the quality control values fell outside of the acceptable ranges.

\section{Diagnostic Accuracy and Prescription Status}

For the 5 drugs/drug classes for which both EIA screen and LC-MS/MS testing were performed, the diagnostic accuracy of the EIA was determined. False negatives were specimens in which EIA was negative and detectable concentrations of drug and/or metabolite were found by LC-MS/MS. True positives were specimens in which EIA was positive and detectable concentrations of corresponding drug(s) and/or metabolite(s) were found by LC-MS/MS. The number of EIA false negative results in patients with and without a prescription for the drug/drug class being tested was determined.

If only LC-MS/MS testing was performed due to lack of a validated EIA method, the total number of true positives was calculated for each drug/drug class. Specimens were then divided into: 1) true positives in patients with prescription for the drug/drug class being tested and 2) true positives in patients without prescription for the drug/drug class being tested.

\section{Results}

Opiates (i.e., hydrocodone, hydromorphone, morphine, and codeine) were the most frequently prescribed drug class in this study, with 284 (53.6\%) patients prescribed an opiate (Fig. 1, Table 2). Two 


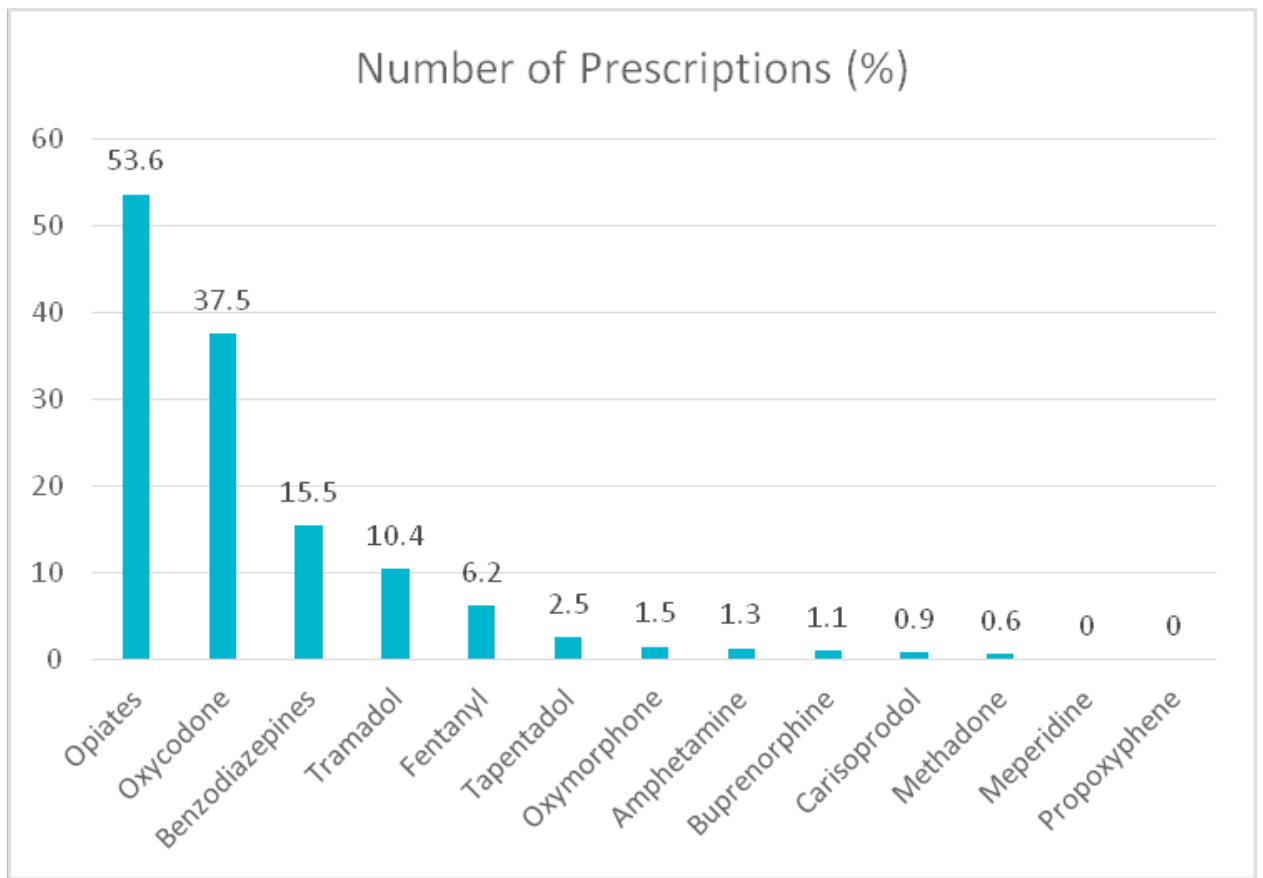

Fig. 1. Percentage of patients with prescriptions for each drug/drug class. Opiate prescriptions include hydrocodone, hydromorphone, morphine, and codeine.

hundred of the 284 (70.4\%) opiate-prescribed patients were prescribed hydrocodone, while prescriptions for morphine, multiple opiates, and hydromorphone made up $18.0 \%, 8.8 \%$, and $2.8 \%$ of opiate-prescriptions, respectively. The other commonly prescribed drugs/drug classes in our patient population were oxycodone or oxymorphone (37.5\% of patients), benzodiazepines $(15.5 \%)$, tramadol $(10.4 \%)$, and fentanyl $(6.2 \%)$ (Fig. 1). The types of benzodiazepines prescribed varied, with $22(26.8 \%), 19(23.2 \%), 17$ (20.7\%), $14(17.1 \%), 4$ $(4.9 \%)$, and $6(7.3 \%)$ patients (of the total of 82 ) being prescribed alprazolam, clonazepam, diazepam, lorazepam, temazepam, and multiple benzodiazepines, respectively.

Table 2 shows the overall frequency of positive LCMS/MS findings for 13 drugs/drug classes. Positive results are also separated by whether the patient had an active prescription for the drug/metabolite detected. An average of 1.6 drugs were detected per patient (831 positive results in 530 patient samples). Approximately one-third of the drugs detected were not prescribed, $32.4 \%(269 / 831$ ) (Table 2). The most frequent unexpected positives (i.e., detected illicit or undisclosed/ non-prescribed drugs) were benzodiazepines, opiates, tramadol, amphetamines, oxycodone/oxymorphone, and carisoprodol. In fact more than half of the positive benzodiazepine $(123 / 185,66.5 \%)$, amphetamine $(20 / 23,87.0 \%)$, buprenorphine $(2 / 3,66.7 \%)$, carisoprodol $(10 / 15,66.7 \%)$, tramadol $(37 / 70,52.9 \%)$, and methadone $(3 / 5,60.0 \%)$ results were detected in patients without a prescription for that drug/metabolite. On the other hand, the majority ( $>80 \%$ ) of detected opiates, oxycodone/oxymorphone, fentanyl, and tapentadol were prescribed (Table 2).

EIA yielded an overall sensitivity of $78.5 \%$, detecting 543 of the 692 LC-MS/MS positives for the 6 drugs/ drug classes (amphetamines, buprenorphine, benzodiazepines, cocaine, opiates, and oxycodone) tested by EIA and LC-MS/MS (Table 3). EIA sensitivity in comparison with LC-MS/MS was less than $80 \%$ for all but one of 6 classes tested. Oxycodone EIA was the exception, detecting $92.5 \%(172 / 186)$ of oxycodone/oxymorphone positive samples. The 2 other most frequently prescribed drug classes, opiates and benzodiazepines, were detected by EIA in only $79.4 \%(228 / 287)$ and $65.4 \%(121 / 185)$ of cases, respectively (Table 3 ). Less than half of the positive buprenorphine $(33.3 \%, 1 / 3)$ and cocaine $(37.5 \%, 3 / 8)$ samples were detected by EIA. 
Immunoassay-Based Drug Tests Are Inadequately Sensitive

Table 2. Numbers and percentages of patients $(N=530)$ with active prescriptions and positive by LC-MS/MS for each prescription drug/drug class.

\begin{tabular}{|c|c|c|c|c|c|c|c|c|}
\hline \multirow[t]{2}{*}{ Drug/Drug Class } & \multicolumn{2}{|c|}{$\begin{array}{c}\text { Number of } \\
\text { Prescriptions }\end{array}$} & \multicolumn{2}{|c|}{ Positive by LC-MS/MS } & \multicolumn{2}{|c|}{$\begin{array}{c}\text { Positive by LC-MS/MS } \\
\text { with Prescription }\end{array}$} & \multicolumn{2}{|c|}{$\begin{array}{c}\text { Positive by LC-MS/MS } \\
\text { without Prescription }\end{array}$} \\
\hline & $\mathbf{N}$ & $\%$ of patients & $\mathbf{N}$ & $\%$ of patients & $\mathbf{N}$ & $\%$ of positives & $\mathbf{N}$ & $\%$ of positives \\
\hline Opiates $^{*}$ & 284 & $53.6 \%$ & 287 & $54.2 \%$ & 249 & $86.8 \%$ & 38 & $13.2 \%$ \\
\hline $\begin{array}{l}\text { Oxycodone/ } \\
\text { Oxymorphone }\end{array}$ & $199 / 8$ & $37.5 \% / 1.5 \%$ & 186 & $35.1 \%$ & 167 & $89.8 \%$ & 19 & $10.2 \%$ \\
\hline Benzodiazepines & 82 & $15.5 \%$ & 185 & $34.9 \%$ & 62 & $33.5 \%$ & 123 & $66.5 \%$ \\
\hline Tramadol & 55 & $10.4 \%$ & 70 & $13.2 \%$ & 33 & $47.1 \%$ & 37 & $52.9 \%$ \\
\hline Fentanyl & 33 & $6.2 \%$ & 38 & $7.2 \%$ & 31 & $81.6 \%$ & 7 & $18.4 \%$ \\
\hline Tapentadol & 13 & $2.5 \%$ & 10 & $1.9 \%$ & 9 & $90.0 \%$ & 1 & $10.0 \%$ \\
\hline Amphetamine & 7 & $1.3 \%$ & 23 & $4.3 \%$ & 3 & $13.0 \%$ & 20 & $87.0 \%$ \\
\hline Buprenorphine & 6 & $1.1 \%$ & 3 & $0.6 \%$ & 1 & $33.3 \%$ & 2 & $66.7 \%$ \\
\hline Carisoprodol & 5 & $0.9 \%$ & 15 & $2.8 \%$ & 5 & $33.3 \%$ & 10 & $66.7 \%$ \\
\hline Methadone & 3 & $0.6 \%$ & 5 & $0.9 \%$ & 2 & $40.0 \%$ & 3 & $60.0 \%$ \\
\hline Meperidine & 0 & $0.0 \%$ & 1 & $0.2 \%$ & 0 & $0.0 \%$ & 1 & $100.0 \%$ \\
\hline Propoxyphene & 0 & $0.0 \%$ & 0 & $0.0 \%$ & 0 & $0.0 \%$ & 0 & $0.0 \%$ \\
\hline Total & 695 & N/A & 831 & N/A & 562 & 67.6 & 269 & 32.4 \\
\hline
\end{tabular}

${ }^{*}$ Includes hydrocodone, hydromorphone, morphine, and codeine.

Positive amphetamines were detected $78.3 \%$ (18/23) of the time by EIA. Overall, EIA missed $21.5 \%$ (149) of LCMS/MS positive results for these 6 drug classes (Table 3). Of the 149 false negative EIA results, nearly half, 69 (46.3\%), were for non-prescribed or illicit drugs.

Fentanyl, meperidine, tramadol, tapentadol, and carisoprodol did not have FDA-cleared EIA methodologies available at the time of this study. Without a specific EIA for each of these drugs, direct LC-MS/MS testing was used for detection. A combined total of 134 (16.1\%) positive carisoprodol (15), fentanyl (38), meperidine (1), tramadol (7), and tapentadol (10) samples were detected by LC-MS/MS. If only patients with an active prescription were tested for these 5 drugs using LC-MS/MS, 58.2\% (78/134) would have been detected; the remaining 56 (41.8\%) were not prescribed (Table 3). Two drugs, methadone and propoxyphene, were not screened by EIA in this study due to the relatively low prevalence of these drugs in this population. It is not known whether available EIAs would have detected the 5 methadone positive samples in this study; no propoxyphene positives were detected.

Use of patients' active prescriptions to determine the need for LC-MS/MS testing would have resulted in an additional 160 drugs (19.3\%) being detected by LC-MS/MS because they would be considered pertinent negatives (i.e., negative or not detected by EIA, but prescribed) (Table 3). An overall sensitivity of $84.6 \%$ (703/831) was achieved by reflexing presumptive EIA positives (543) plus pertinent negatives (160) to LC-MS/ MS. However, with this testing algorithm, 128 positive drugs (15.4\%) would have been missed (Table 3). Benzodiazepines and tramadol made up the large majority of false negatives, with 40 and 37 positives missed, respectively. Of the 40 benzodiazepines missed by this algorithm, 17 (42.5\%) were lorazepam, 15 (37.5\%) were 7-aminoclonazepam (clonazepam metabolite), and the remaining 8 were alpha-hydroxyalprazolam $(n=3)$ or combinations of oxazepam, temazepam, and/or nordiazepam $(n=5)$. ElA did not detect any of the patients with undisclosed lorazepam use. The opiates and other opioids accounted for 31 of the false negatives (opiates $=13$, fentanyl $=7$, oxycodone/oxymorphone $=5$, methadone $=3$, buprenorphine $=1$, meperidine $=1$, tapentadol $=1$ ). Of the 13 opiates that were missed, 12 were positive for hydrocodone and one was positive for morphine. Of the 35 instances of undisclosed hydrocodone use detected by LC-MS/MS, only $23(65.7 \%)$ were detected by EIA.

If the same protocol is applied only to the 6 drugs/ drug classes tested by both EIA and LC-MS/MS, 10\% (69/692) of drugs would not have been detected, because they screened negative and were not prescribed (Table 3). Both illicit amphetamine/methamphetamine 
Table 3. Total samples positive for each drug/drug class by LC-MS/MS along with corresponding number of EIA true positives, pertinent negatives (prescribed drugs positive by LC-MS/MS but not detected by EIA), and overall false negatives (total- [EIA positives + pertinent negatives]) are presented. The EIA and overall (combining EIA and pertinent negatives) sensitivities are also presented. Drugs/drug classes not tested by EIA are represented by '--'.

\begin{tabular}{|c|c|c|c|c|c|c|}
\hline $\begin{array}{l}\text { Drug/Drug } \\
\text { Class }\end{array}$ & Total & $\begin{array}{l}\text { EIA True } \\
\text { Positives }\end{array}$ & $\begin{array}{l}\text { Pertinent } \\
\text { Negatives }\end{array}$ & $\begin{array}{c}\text { Overall False } \\
\text { Negatives }\end{array}$ & EIA Sensitivity & $\begin{array}{c}\text { Overall } \\
\text { Sensitivity }\end{array}$ \\
\hline Opiates* & 287 & 228 & 46 & 13 & $79.4 \%$ & $95.5 \%$ \\
\hline $\begin{array}{l}\text { Oxycodone/ } \\
\text { Oxymorphone }\end{array}$ & 186 & 172 & 9 & 5 & $92.5 \%$ & $97.3 \%$ \\
\hline Benzodiazepines & 185 & 121 & 24 & 40 & $65.4 \%$ & $78.4 \%$ \\
\hline Tramadol & 70 & -- & 33 & 37 & -- & $47.1 \%$ \\
\hline Fentanyl & 38 & -- & 31 & 7 & -- & $81.6 \%$ \\
\hline Amphetamines & 23 & 18 & 0 & 5 & $78.3 \%$ & $78.3 \%$ \\
\hline Carisoprodol & 15 & -- & 5 & 10 & -- & $33.3 \%$ \\
\hline Tapentadol & 10 & -- & 9 & 1 & -- & $90.0 \%$ \\
\hline Cocaine & 8 & 3 & 0 & 5 & $37.5 \%$ & $37.5 \%$ \\
\hline Methadone & 5 & -- & 2 & 3 & -- & $40.0 \%$ \\
\hline Buprenorphine & 3 & 1 & 1 & 1 & $33.3 \%$ & $66.7 \%$ \\
\hline Meperidine & 1 & -- & 0 & 1 & -- & $0.0 \%$ \\
\hline Propoxyphene & 0 & -- & 0 & 0 & -- & N/A \\
\hline Total & 831 & 543 & 160 & 128 & 78.5 & 84.6\% \\
\hline
\end{tabular}

${ }^{*}$ Includes hydrocodone, hydromorphone, morphine, and codeine.

and cocaine use would have been missed in $5(1 \%)$ of the population. Based on this data, one out of $10 \mathrm{pa}-$ tients using undisclosed and/or illicit drugs would have been missed using EIA plus prescription status to determine the need for LC-MS/MS confirmation testing.

Of the 530 patients tested, 4 had high concentrations of parent drug and no corresponding metabolite by LC-MS/MS, and were suspected of simulating compliance; 2 with oxycodone and 2 with hydrocodone. In these patients, high levels of the parent compound (i.e., 3 cases of oxycodone and hydrocodone $>10,000 \mathrm{ng} / \mathrm{mL}$ and one case of hydrocodone of $1211 \mathrm{ng} / \mathrm{mL}$ ) were present in the urine but no metabolites (i.e., oxymorphone/ noroxycodone and hydromorphone/norhydrocodone, respectively) were detected. This is most likely due to the patients adding medication directly into their urine in an attempt to simulate compliance.

Urine creatinine fell outside the reference range of $20-300 \mathrm{mg} / \mathrm{dL}$ in $7.2 \%$ of patients. Sixteen patients $(3.0 \%)$ had a creatinine $<20 \mathrm{mg} / \mathrm{dL}$; this indicates a dilute urine and suggests possible adulteration of the sample. Another 22 patients had urine creatinine $>300$ $\mathrm{mg} / \mathrm{dL}$; this indicates a very concentrated specimen that may interfere with absorbance readings in EIA, but not LC-MS/MS.

\section{Discussion}

EIAs revolutionized drug testing in the 1980s. Providing rapid turn-around times and the ability to simplify testing on automated platforms, EIAs allow even small, community hospitals access to drug testing results 24 hours a day and 7 days a week. These tests are routinely used for drug monitoring in hospitalized patients, determining drug(s) used in overdose cases, and the federal workplace drug testing program. However, all of the current, commercially available FDA-cleared EIAs used for pain management have not been evaluated by the FDA for use in this unique population. Limitations, most notably, lack of sensitivity and poor detection of new drugs have prompted the need for alternative testing in this population.

When a clinician is attempting to confirm adherence with a prescribed treatment plan, the sensitivity of the drug test is often more important than the specificity. False positives due to lack of specificity are easily avoided by following up with a highly specific confirmatory method. However, false negatives frequently go undetected, creating a greater risk of misdirected therapy. Supplementing or altogether bypassing the EIA screens and proceeding directly to LC-MS/ 
MS is recommended to solve the sensitivity challenges of EIA in the pain management population. LC-MS/ MS provides highly sensitive and specific detection of both parent drugs and metabolites, differentiation of multiple drugs within a class, and detection capabilities for additional drug/drug classes that do not have available EIAs. Limiting confirmatory/quantitative testing to only EIA positives and pertinent negatives puts patients and doctors at unnecessary risk as undisclosed use and abuse of many drugs and medications would be missed (27).

Similar to published studies, we demonstrate a high rate of false negatives, $21.5 \%$, using EIA to screen samples originating from patients treated for chronic pain $(15,19,23-25)$. The largest number of EIA false negatives were for benzodiazepines, followed by opiates, oxycodone, amphetamines, cocaine, and buprenorphine (Table 3). More than $20 \%$ of each of these drugs were missed by EIA, with the exception of oxycodone which was detected in $92.5 \%$ of cases. More than $50 \%$ of positive cocaine and buprenorphine samples were missed by EIA. These findings suggest that EIA should not be used to definitively test for amphetamines, buprenorphine, benzodiazepines, cocaine, opiates, and oxycodone in the pain management population. False negative EIA results were particularly common in patients using lorazepam, clonazepam, and hydrocodone. EIA screens were not performed for carisoprodol, fentanyl, meperidine, methadone, propoxyphene, tapentadol, and tramadol, several of which have commercially available immunoassays. Therefore sensitivity of these screens compared to LC-MS/MS was not assessed.

Confirmation of pertinent negatives provided only a marginal increase in sensitivity because of the prevalence of undisclosed drug use. Adding confirmation of prescribed drugs that test negative by EIA (pertinent negatives), in addition to the EIA positives, increased sensitivity to $84.6 \%$, but still left more than $10 \%$ of patients using undisclosed and/or illicit drugs undetected. Approximately one-third of all drugs detected by LCMS/MS in this study (32.4\%) were not prescribed. Benzodiazepines, opiates, tramadol, amphetamines, oxycodone, and carisoprodol were most frequently present without a prescription. Pertinent negative confirmation did not improve sensitivity of illicit drugs like cocaine because they are not prescribed. It is important to note that $57(11 \%)$ of our patients had no medication history provided on the requisition indicating that the patient was not prescribed pertinent medications or the provider failed to fill out the requisition completely. In the lat- ter case, we would inaccurately classify these patients as being prescribed no medications and the number of false negative patients without a prescription could be overestimated.

As an alternative to prioritizing LC-MS/MS testing, EIA cutoffs could be lowered to increase sensitivity and reduce EIA false negatives, particularly for buprenorphine, cocaine, and amphetamine, for example, for which lower cutoffs are readily available on some platforms. However this option presents challenges. For one, cutoff adjustments with point-of-care urine cups may not be an option. If adjustment is possible, lowering EIA cutoffs to improve EIA sensitivity complicates the interpretation by producing higher rates of false positive results. Extensive education and experience is required to accurately interpret information obtained from EIA because the assays are often inaccurate and incomplete. Increasing the frequency of false positive EIA results further increases the risk of inappropriate result interpretation and subsequent patient harm (e.g., inappropriate dismissal from the program). Adjusting cutoffs away from the manufacturers' recommendations also increases complexity of the testing, making the tests lab developed tests (LDTs).

EIA performance was not evaluated for tramadol, fentanyl, carisoprodol, tapentadol, methadone, meperidine, and propoxyphene as EIA screening for these drugs/drug classes was not performed. However, we did find a high number of positive LC-MS/MS results for these drugs in patients without a prescription, suggesting the importance of a sensitive and specific technique to assess compliance. Two exceptions, meperidine and propoxyphene, neither of which are frequently abused or prescribed, could potentially be removed from any customized test profiles if prevalence remains low.

Identification of urine spiking and/or dilution is important for compliance monitoring. Patients diverting the medication for financial or other reasons, have incentive to simulate compliance by either adding the drug directly to their urine or taking large quantities just prior to their office visit. We identified $4(0.8 \%)$ patients who appeared to simulate compliance by placing the drug directly in their urine because only the parent compound was present at high levels by LC-MS/MS and not the metabolite(s). This illustrates another benefit of LC-MS/MS testing, because without the LC-MS/ MS results, the provider may have incorrectly assumed compliance.

Creatinine has long been used to recognize diluted or substituted specimens; $3.0 \%$ of patients in our study 
had unusually dilute urine suggestive of adulteration. In addition to providing information regarding sample integrity/validity, creatinine also plays an important role in allowing normalization of drug results based on the patient's hydration status and can allow for trending of positive results for drugs that may remain in a patient's system for an extended period of time. We recommend continuing to measure creatinine in conjunction with UDT in pain management as it provides valuable information.

Poly-pharmacy is the largest contributor to drug related deaths $(28,29)$. Therefore, it is extremely important to identify unknown medications or illicit compounds in an effort to reduce the potential for lethal interactions with prescribed medications. Our findings support recommendations from experts in the field of pain management testing who assert, "if a choice is possible, it is desirable to use an assay with well-documented specificity at the lowest threshold or cutoff possible" (30). Our study demonstrates that LC-MS/MS offers far superior sensitivity for medication monitoring in the pain management population. Even using more sensitive LC-MS/MS, reportable limits must be carefully evaluated to ensure detection of compounds that are frequently present at lower concentrations such as buprenorphine/norbuprenorphine and fentanyl.

Due to the high volume of false negative EIA results in patients with and without prescriptions, the high volume of positive results that reflex to LC-MS/ MS, and/or the high number of providers prescribing these medications, LC-MS/MS should be performed in addition to or instead of EIA screening. LC-MS/MS can measure more than 50 drugs in a single injection using as little as $1 \mathrm{~mL}$ of sample, and offers customizable drug targets and cutoffs, high-accuracy quantitative results, and metabolite information. LC-MS/MS is also amenable to customized reporting with automatic interpretations of the presence and/or absence of drugs as consistent or inconsistent with the patients prescribed therapy, enabling rapid and accurate interpretation of toxicology results. Drug testing should be performed randomly, with a maximum frequency determined, and comprehensively (including all frequently abused/diverted drugs in the population of patients being monitored) to avoid dangerous interactions.

Cost-benefit analysis and risk stratification are necessary in deciding frequency of drug test monitoring. The acceptance of direct to definitive testing as the best clinical practice in monitoring patients treated for chronic pain means an increased cost of testing. LC-MS/ MS testing is more technically challenging compared to EIA, necessitating higher level personnel to perform and interpret testing. The reagent, lease, and service costs for EIA and LC-MS/MS are variable and dependent on many factors including test volume, test platform (e.g., point of care versus laboratory-based) and laboratory infrastructure (e.g., existing equipment). Although a larger investment is required for LC-MS/MS-based testing, and there is an increased cost of testing, this may be outweighed by the risk and cost of failing to detect non-adherence and drug abuse for the patient, health care professional, and for society. Limiting the extent of testing (ex. excluding certain prescribed or illicit drugs/classes) or utilizing inferior technologies such as EIA (inferior sensitivity and specificity compared to MS-based techniques) to determine risk of opioid and other drug misuse can place both the provider and patient at risk. Many of the current payment models do not support the use of LCMS/MS testing for all patients. It is viewed as too expensive for many government and private payers to reimburse laboratories for large definitive testing panels under the established payment structures. As a solution, payers should be encouraged to provide reasonable and sustainable payment models for preferred (definitive) testing, rather than forcing the use of inferior and inaccurate testing methods on providers.

\section{References}

1. Harrell A. The validity of self-reported drug use data: The accuracy of responses on confidential self-adminstered answered sheets. NIDA Res Monogr 1997; 167:37-58.

2. Lesler J, O'Rielly J. Mode of interview and reporting of sensitive issues: Design and implementation of audio comput- er-assisted self-interviewing. NIDA Res Monogr 1997; 167:366-382.

3. Harrison L. The validity of self-reported drug use: Improving the accuracy of survey estimates. NIDA Res Monogr 1997; 167:17-36.

4. Skala K, Reichl L, Ilias W, Likar R, Grogl-Aringer G, Wallner C, Schlaff G, Her- rmann $\mathrm{P}$, Lesch $\mathrm{O}$, Walter $\mathrm{H}$. Can we predict addiction to opioid analgesics? A possible tool to estimate the risk of opioid addiction in patients with pain. Pain Physician 2013; 16:593-601.

5. Hamill-Ruth RJ, Larriviere K, McMasters MG. Addition of objective data to identify risk for medication misuse and abuse: 
The inconsistency score. Pain Med 2013; 14:1900-1907.

6. Cone EJ, Caplan YH, Black DL, Robert T, Moser F. Urine drug testing of chronic pain patients: Licit and illicit drug patterns. J Anal Toxicol 2008; 32:530-543.

7. Katz NP, Sherburne S, Beach M, Rose RJ, Vielguth J, Bradley J, Fanciullo GJ. Behavioral monitoring and urine toxicology testing in patients receiving longterm opioid therapy. Anesth Analg 2003; 97:1097-1102.

8. Melanson SE, Kredlow MI, Jarolim P. Analysis and interpretation of drug testing results from patients on chronic pain therapy: A clinical laboratory perspective. Clin Chem Lab Med 2009; 47:971-976.

9. Michna E, Jamison RN, Pham LD, Ross EL, Janfaza D, Nedeljkovic SS, Narang S, Palombi D, Wasan AD. Urine toxicology screening among chronic pain patients on opioid therapy: Frequency and predictability of abnormal findings. Clin J Pain 2007; 23:173-179.

10. Magnani B, Kwong T. Urine drug testing for pain management. Clin Lab Med 2012; 32:379-290.

11. Heit HA, Gourlay DL. Urine drug testing in pain medicine.J Pain Symptom Manage 2004; 27:260-267.

12. Ives TJ, Chelminski PR, Hammett-Stabler CA, Malone RM, Perhac JS, Potisek NM, Shilliday BB, DeWalt DA, Pignone MP. Predictors of opioid misuse in patients with chronic pain: A prospective cohort study. BMC Health Serv Res 2006; 6:46.

13. Nafziger AN, Bertino, Jr. JS. Utility and application of urine drug testing in chronic pain management with opioids. Clin J Pain 2009; 25:73-79.

14. Manchikanti L, Atluri S, Trescot AM, Giordano J. Monitoring opioid adherence in chronic pain patients: Tools, techniques, and utility. Pain Physician 2008; 11:S155-S180.
15. Darragh A, Snyder ML, Ptolemy AS, Melanson S. KIMS, CEDIA, and HSCEDIA immunoassays are inadequately sensitive for detection of benzodiazepines in urine from patients treated for chronic pain. Pain Physician 2014; 17:359366.

16. Bottcher M, Beck O. Evaluation of buprenorphine CEDIA assay versus GCMS and ELISA using urine samples from patients in substitution treatment. J Anal Toxicol 2005; 29:769-776.

17. Mikel C, Pesce AJ, Rosenthal M, West C. Therapeutic monitoring of benzodiazepines in the management of pain: Current limitations of point of care immunoassays suggest testing by mass spectrometry to assure accuracy and improve patient safety. Clin Chim Acta 2012; 413:1199-1202.

18. Pavlic $M$, Libiseller $K$, Grubwieser $P$, Rabl W. Cross-reactivity of the CEDIA buprenorphine assay with opiates: An austrian phenomenon? Int J Legal Med 2005; 119:378-381.

19. Pesce A, Rosenthal M, West R, West $C$, Crews B, Mikel C, Almazan P, Latyshev $\mathrm{S}$. An evaluation of the diagnostic accuracy of liquid chromatography-tandem mass spectrometry versus immunoassay drug testing in pain patients. Pain Physician 2010; 13:273-281.

20. Glover SJ, Allen KR. Measurement of benzodiazepines in urine by liquid chromatography-tandem mass spectrometry: Confirmation of samples screened by immunoassay. Ann Clin Biochem 2010; 47:111-117.

21. Melanson SE. The utility of immunoassays for urine drug testing. Clin Lab Med 2012; 32:429-447.

22. Lu NT, Taylor BG. Drug screening and confirmation by GC-MS: Comparison of EMIT II and Online KIMS against 10 drugs between US and England laboratories. Forensic Sci Int 2006; 157:106-116.
23. Mikel C, Almazan P, West R, Crews B, Latyshev S, Pesce A, West C. LC-MS/ MS extends the range of drug analysis in pain patients. Ther Drug Monit 2009; 31:746-748.

24. Melanson SE, Snyder ML, Jarolim P, Flood JG. A new highly specific buprenorphine immunoassay for monitoring buprenorphine compliance and abuse. J Anal Toxicol 2012; 36:201-206.

25. Manchikanti L, Malla Y, Wargo BW, Fellows B. Comparative evaluation of the accuracy of benzodiazepine testing in chronic pain patients utilizing immunoassay with liquid chromatography tandem mass spectrometry (LC/MS/MS) of urine drug testing. Pain Physician 2011; 14:259-270.

26. Palmetto GBA, Local Coverage Determination (LCD): Controlled Substance Monitoring and Drugs of Abuse Testing (L35105), Contract Number 11202, copyright 2002-2014, American Medical Association.

27. Fitzgibbon DR, Rathmell JP, Michna E, Stephens LS, Posner KL, Domino KB. Malpractice claims associated with medication management for chronic pain. Anesthesiology 2010; 112:948-956.

28. Administration, Substance Abuse and Mental Health Service. The DAWN report: Outcomes of drug-related emergency department visits associated with polydrug use. Rockville, MD: 2012 July 12.

29. Jones JD, Mogali S, Comer SD. Polydrug abuse: A review of opioid and benzodiazepine combination use. Drug Alcohol Depend 2012; 125:8-18.

30. Hammett-Stabler C, Magnani B. Supporting the pain service. In: Magnani B, Bissell MG, Kwong TC, Wu AH (eds). Clinical Toxicology: A Guide for Laboratory Professionals. CAP Press, Northfield, IL, 2012, pp 15-26. 
